\title{
Dynamic modelling and visco-elastic parameter identification of a fibre-reinforced soft fluidic elastomer manipulator
}

\author{
Azadeh Shariati, Jialei Shi, Sarah Spurgeon and Helge A Wurdemann
}

\begin{abstract}
A dynamic model of a soft fibre-reinforced fluidic elastomer is presented and experimentally verified, which can be used for model-based controller design. Due to the inherent visco-(hyper)elastic characteristics and nonlinear timedependent behaviour of soft fluidic elastomer robots, analytic dynamic modelling is challenging. The fibre reinforced noninflatable soft fluidic elastomer robot used in this paper can produce both planar and spatial movements. Dynamic equations are developed for both cases. Parameters, related to the viscoelastic behaviour of the robot during elongation and bending motion, are identified experimentally and incorporated into our model. The modified dynamic model is then validated in experiments comparing the time responses of the physical robot with the corresponding outputs of the simulation model. The results validate the accuracy of the proposed dynamic model.
\end{abstract}

\section{INTRODUCTION}

Continuum robotic manipulators have been inspired by octopus arms [1], elephant trunks [2]-[4], eels [5], and snakes [6] able to bend and navigate around obstacles. As most continuum robots are extrinsically actuated, studies on modelling continuum robots have mainly focussed on kinematic and dynamic modelling of tendon-driven continuum robots [2], [7]. Such models have been very important in developing control systems for continuum robots [8]. Gravagne and Walker conducted one of the earliest studies on the dynamic behaviour of continuum robots [3] deriving equations for 2-dimensional planar motion. In [9], Kapadia and Walker advanced this work and presented a model-based dynamic controller in the task-space. This dynamic model is obtained using the Euler-Lagrange approach. Experimental validation indicates that an inverse dynamics-based PD control system converges despite uncertainties in the model parameters. Again, this model and controller is based on 2D planar motion. In [10], [11], the Lagrange approach was also used for dynamic modelling of a tendon-driven robot. The theoretical model considers planar dynamic behaviour of a continuum manipulator validated using simulation only.

Soft material robotics has become an important research topic [12], [13] aiming at a wide range of applications [14][16]. Many fluidic-driven soft robots are made of elastomer materials, which exhibit visco-elastic behaviour, which needs

\footnotetext{
*This work is supported by the Springboard Award of the Academy of Medical Sciences (grant number: SBF003-1109) and the Engineering and Physical Sciences Research Council (grant numbers: EP/R037795/1, $\mathrm{EP} / \mathrm{S} 014039 / 1$ and EP/V01062X/1).

A. Shariati, J. Shi and H. Wurdemann are with the Department of Mechanical Engineering, University College London, WC1E 7JE, UK. h.wurdemann@ucl.ac.uk, a.shariati@ucl.ac.uk

Sarah Spurgeon is with the Department of Electronic and Electrical Engineering, University College London, WC1E 7JE, UK. s.spurgeon@ucl.ac.uk
}

to be incorporated in the model. A number of developments have resulted in the field of soft robotics including advancements in modelling soft continuum robots [17]-[19]. In [20], a machine learning-based method is introduced for modelling the dynamics of a tendon-driven soft robot. Further research focussed on using a recurrent neural network to obtain a dynamic model and develop a closed-loop predictive controller based on a learning algorithm [21]. The model is limited to tendon-driven soft robots and it is based on learning algorithms (model-free techniques) rather than model-based dynamics. Focussing on fluidic-driven bellow robots, dynamic equations for the Bionic Handling Assistant Robot, a 3-section continuum robot, have been derived and validated for 3D motion [22], [23]. A dynamic model-based controller was subsequently developed, specifically, for the bellow robot [24], [25].

Research on dynamic modelling and real-time control of reinforced soft fluidic elastomer robots is limited [26] [28]. Marchese et al. derived a dynamic model of a soft manipulator with four segments made of elastomer with fluidically actuated chambers. They developed a locally optimal open-loop controller to grasp objects and perform dynamic manoeuvres [26]. Santina, Katzschmann and coworkers worked on the same soft fluidic elastomer robot. Their formulation relates the dynamic response of the soft robot to a rigid-body manipulator and experiments validate the performance of control of bending [29], [30]. In both research studies, the dynamic equations for each section were obtained considering one Degree-of-Freedom (DoF), i.e., one variable of planar bending is considered and elongation and spatial bending is neglected in the modelling and control algorithms. Onal and Rus presented dynamic equations and control design of an undulatory soft robot. The dynamic modelling and control of this robot includes the control of planar bending only for each of the four segments [31]. Mustaza et al. developed a nonlinear dynamic model for a soft fluidic elastomer robot based on a composite material model [28], [32]. Dynamic modelling of a reinforced soft fluidic elastomer robot is investigated and a visco-hyperelastic material based continuum approach is considered. The contribution focuses on the derivation of the energy term relating to the elasticity and viscosity of the manipulator. Important details of the kinetic energy in the Lagrange equation, which describes the motion of the manipulator, are omitted.

Overall, extensive work has been conducted on kinematic and dynamic modelling for (tendon-driven) continuum robotic manipulators. Investigating both model-free [21] and model-based dynamics [26] for soft fluidic elastomer robots 
has been less explored. In particular, one of the benefits of model-based dynamics is their transferability across different designs by adjusting geometrical, inertial and other parameters associated with the material such as visco-elastic parameters. Whereas geometrical and inertial parameters can be easily measured, identifying visco-elastic parameters using an analytical approach can be challenging [28].

The contribution of this paper lies in the system identification for a soft robotic dynamical model that describes spatial/planar movements. In particular, we introduce (longitudinal/bending damping, planar bending stiffness/damping, spatial bending stiffness/damping) visco-elastic coefficients resulting from the response of the soft material when deformed, e.g., through fluidic actuation. A modified version of the STIFF-FLOP manipulator is created [33], allowing for individual actuation of each of the six reinforced chambers (Fig. 2), for system identification and validation.

The design and manufacturing process for the modified version of the fibre-reinforced non-inflatable soft fluidic elastomer robot is presented in Section II. In Section III, the prerequisite kinematics needed for the dynamical modelling is described. In Section IV, the nonlinear dynamic equations of the soft robot are presented for two general cases: (1) spatial motion with three pneumatic inputs and (2) planar motion with two pneumatic inputs. These dynamic equations are extended in Section IV to include the response of the fluidic elastomer. The corresponding visco-elastic parameters associated with this specific class of soft fluidic elastomer robot are identified in Section V. The dynamic model is validated by comparing the time response of the robot to different actuation inputs in experiments and simulation.

\section{FABRICATION PROCESS OF OUR SOFT FLUIDIC ELASTOMER MANIPULATOR}

The soft fluidic elastomer manipulator used in this research study is a modified version of the STIFF-FLOP manipulator [33] (see Fig. 1). One of the most important design elements of this soft robot lie in the fabrication process which results in individually reinforced actuation chambers producing elongation only when pressurised. The original STIFF-FLOP manipulator is modified by extending the length to $110 \mathrm{~mm}$ and allowing each chamber to be individually actuated. (Note: The original length is $55 \mathrm{~mm}$. Two actuation chambers were connected resulting in three chamber pairs.) The new design provides an opportunity to change the arrangement of pneumatic input patterns and produce different planar or spatial motions.

The fabrication process is shown in Fig. 1. Each chamber is individually reinforced by a helical thread wrapped around a rod to constrain radial inflation of the chamber when pressurised and, hence, to allow longitudinal expansion only (see Fig. 1(a)). Six braided rods are embedded into a cylindrical mould filled in with Ecoflex ${ }^{\mathrm{TM}}$ 00-50. Once the rods are removed and the fibre threads remain inside the silicone material, rods with a smaller diameter are inserted (see Fig. 1 (b)). Ecoflex ${ }^{\mathrm{TM}}$ 00-30 material is used to fill the gap between these smaller rods and the threads. In a final step, the bottom and top cap are moulded using Dragon Skin ${ }^{\mathrm{TM}} 20$ elastomer (see Fig. 1 (c)). Six pipes are connected to the six chambers to supply pressurised air actuation. The chamber arrangements and dimensions of the soft manipulator are summarised in Fig. 2(a). As all chambers are symmetrically arranged and equally distributed along the central axis, it is possible to apply pneumatic pressure in different ways to achieve planar and spatial motion as illustrated in Fig. 2(b).

\section{DERIVING THE KINEMATIC MODEL}

A well-known and widely used method for kinematic modelling of continuum robots is the Piecewise Constant Curvature approach and this is applied here [7]. The actuator space (length of the tendons/cables, i.e., $\left[l_{1}, l_{2}, l_{3}\right]$ ), configuration space (arc parameters, i.e., $[L, \theta, \phi]$ ) and task space (position and orientation) have been defined to describe the overall pose. The kinematic calculations are in the configuration space, i.e., returning $[L, \theta, \phi]$. For the kinematic analysis, the variables shown in Fig. 2 are used. Frame $X_{w}, Y_{w}, Z_{w}$ is the base reference frame. Frame $X_{h}, Y_{h}, Z_{h}$ is located at the tip of the robot. The matrix in Equation (1) describes the transformation between these frames.

$$
{ }^{w} T_{h}=\left[\begin{array}{ccc}
\cos ^{2} \phi(\cos \theta-1)+1 & \sin \phi \cos \phi(\cos \theta-1) \\
\sin \phi \cos \phi(\cos \theta-1) & \cos ^{2} \phi(1-\cos \theta)+\cos \theta \\
-\cos \phi \sin \theta & -\sin \phi \sin \theta \\
0 & 0 \\
& \cos \phi \sin \theta & L \cos \phi \frac{(1-\cos \theta)}{\theta} \\
& \cos \phi \sin \theta & L \sin \phi \frac{(1-\cos \theta)}{\theta} \\
\cos \theta & L \frac{\sin \theta}{\theta} \\
0 & 1
\end{array}\right]
$$

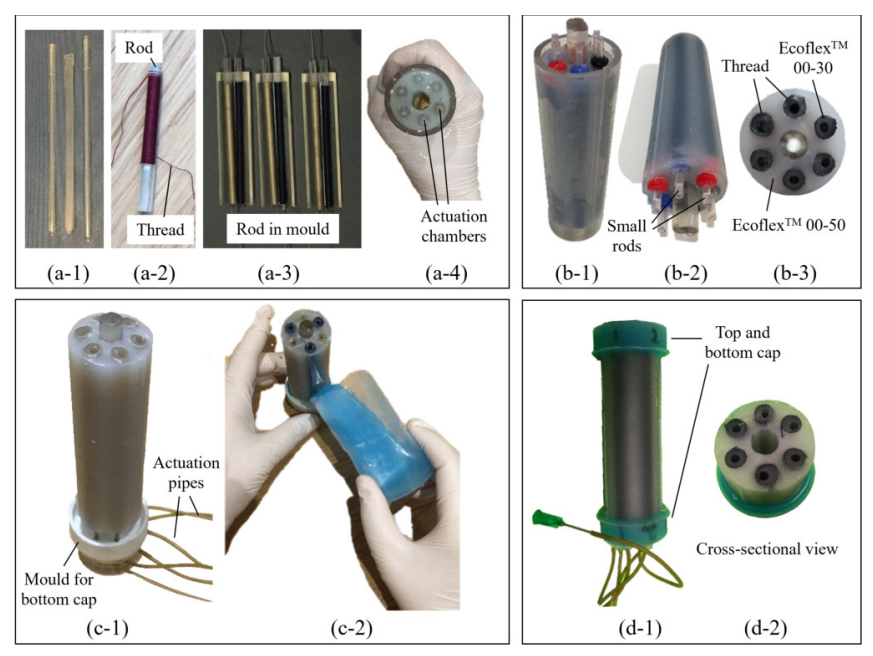

Fig. 1. Fabrication process: (a) First moulding stage: (a-1) A rod sliced into three parts is used for each of the six actuation chambers. (a-2) A fabric thread is wrapped around each rod for axial reinforcement. (a-3) Six rods are prepared and (a-4) equally distributed around the central axis inside the mould, filled with Ecoflex ${ }^{\mathrm{TM}}$ 00-50. (b) Second moulding stage: After removing initial rods, smaller rods are inserted into actuation chambers, filled with Ecoflex ${ }^{\mathrm{TM}}$ 00-30. (c) Third moulding stage: The top and bottom (green colour) is moulded using Dragon Skin ${ }^{\mathrm{TM}} 20$ elastomer. A pipe is connected for each of the six actuation chambers. (d) The final configuration of the soft fluidic elastomer robot and a cross-sectional view. 
The variables $\theta$ and $\phi$ are the planar and spatial bending angles and are shown in Fig. 2(a). Variable $L$ is the default length of the manipulator plus the elongation due to the applied pressure $L=L_{0}+d$. The subscript $h$ refers to the frame located at the tip of the robot and superscript $w$ to the base reference frame. The displacement $X_{T}$ of the manipulator's tip is represented by the last column of matrix ${ }^{w} T_{h}$ in Equation (2).

$$
X_{T}=L\left[\cos \phi \frac{(1-\cos \theta)}{\theta}, \sin \phi \frac{(1-\cos \theta)}{\theta}, \frac{\sin \theta}{\theta}\right]^{T}
$$

The constant curvature assumption can cause singularities in the kinematic model and resulting dynamics. Godage solved this problem suggesting a new shape function [34]. The Taylor expansion method is used here to avoid singularities when $\theta=0$ resulting in Equation (2). It is important to note that the first five terms of the Taylor expansion is used in this paper.

$$
X_{T}=L\left[\cos \phi \sum_{n=1}^{\infty} \frac{(-1)^{n+1} \theta^{2 n-1}}{(2 n) !}, \sin \phi \sum_{n=1}^{\infty} \frac{(-1)^{n+1} \theta^{2 n-1}}{(2 n) !}, \sum_{n=0}^{\infty} \frac{(-1)^{n} \theta^{2 n}}{(2 n+1) !}\right]^{T}
$$

Considering $\dot{X_{T}}=J \dot{q}$, where $X_{T}=\left[x_{T}, y_{T}, z_{T}\right]^{T}$ is the task space vector, $q=[L, \theta, \phi]^{T}$ and $J$ is the Jacobian. Computing the first derivative of the transformation matrix in Equation (1) with respect to time, the velocity of the manipulator's tip is described in the last column of matrix ${ }^{w} \dot{T}_{h}$ resulting in:

$\dot{x}_{T}=(\dot{L} \cos \phi-L \dot{\phi} \sin \phi) \sum_{n=1}^{\infty} \frac{(-1)^{n+1} \theta^{2 n-1}}{(2 n) !}+L \dot{\theta} \cos \phi \sum_{n=1}^{\infty} \frac{\left((-1)^{n+1} \theta^{2 n-2}\right)}{(2 n)(2 n-2) !}$

$\dot{y}_{T}=(\dot{L} \sin \phi+L \dot{\phi} \cos \phi) \sum_{n=1}^{\infty} \frac{(-1)^{n+1} \theta^{2 n-1}}{(2 n) !}+L \dot{\theta} \sin \phi \sum_{n=1}^{\infty} \frac{(-1)^{n+1} \theta^{2 n-2}}{(2 n)(2 n-2) !}$

$\dot{z}_{T}=\dot{L} \sum_{n=0}^{\infty} \frac{(-1)^{n} \theta^{2 n}}{(2 n) !}+L \dot{\theta} \sum_{n=0}^{\infty} \frac{(-1)^{n} \theta^{2 n-1}}{(2 n+1)(2 n-1) !}$

\section{DYNAMIC MODELLING}

In this section, the dynamic equations are obtained using the Lagrange approach:

$$
\frac{d}{d t}\left(\frac{\partial \mathscr{L}}{\partial \dot{q}_{i}}\right)-\frac{\partial \mathscr{L}}{\partial q_{i}}=Q_{i}, i=1, . ., N
$$

where $\mathscr{L}$ is the Lagrangian, equal $K-U$. Variables $K$, $U, q_{i}, Q_{i}$, and $N$ refer to the kinetic and potential energy, generalised coordinates and force, as well as the number of coordinates. Computing linear and angular velocity vectors, the total kinetic energy of the system is acquired as $K_{\text {Total }}=$ $\frac{1}{2} m{\dot{X_{T}}}^{T} \dot{X}_{T}+\frac{1}{2} I \omega^{T} \omega$. As the kinetic term relating to the angular velocity is neglectable [24], the kinetic energy yields:

$$
\begin{aligned}
K= & \frac{1}{2} m \dot{L}^{2}\left(\left(\sum_{n=1}^{\infty} \frac{(-1)^{n+1} \theta^{2 n-1}}{(2 n) !}\right)^{2}+\left(\sum_{n=0}^{\infty} \frac{(-1)^{n} \theta^{2 n}}{(2 n) !}\right)^{2}\right)+ \\
& \frac{1}{2} m L^{2} \dot{\phi}^{2}\left(\sum_{n=1}^{\infty} \frac{(-1)^{n+1} \theta^{2 n-1}}{(2 n) !}\right)^{2}+ \\
& \frac{1}{2} m L^{2} \dot{\theta}^{2}\left(\left(\sum_{n=1}^{\infty} \frac{(-1)^{n+1} \theta^{2 n-2}}{(2 n)(2 n-2) !}\right)^{2}+\left(\sum_{n=0}^{\infty} \frac{(-1)^{n} \theta^{2 n-1}}{(2 n+1)(2 n-1) !}\right)^{2}\right)+ \\
& m L \dot{L} \dot{\theta}\left(\sum_{n=1}^{\infty} \frac{(-1)^{n+1} \theta^{2 n-2}}{(2 n)(2 n-2) !}\right)\left(\sum_{n=1}^{\infty} \frac{(-1)^{n+1} \theta^{2 n-1}}{(2 n) !}\right)+ \\
& m L \dot{L} \dot{\theta}\left(\sum_{n=0}^{\infty} \frac{(-1)^{n} \theta^{2 n}}{(2 n) !}\right)\left(\sum_{n=0}^{\infty} \frac{(-1)^{n} \theta^{2 n-1}}{(2 n)(2 n-1) !}\right)
\end{aligned}
$$
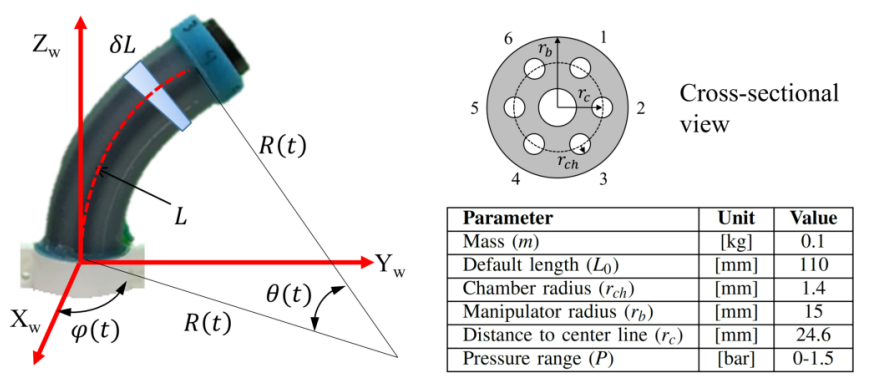

(a)

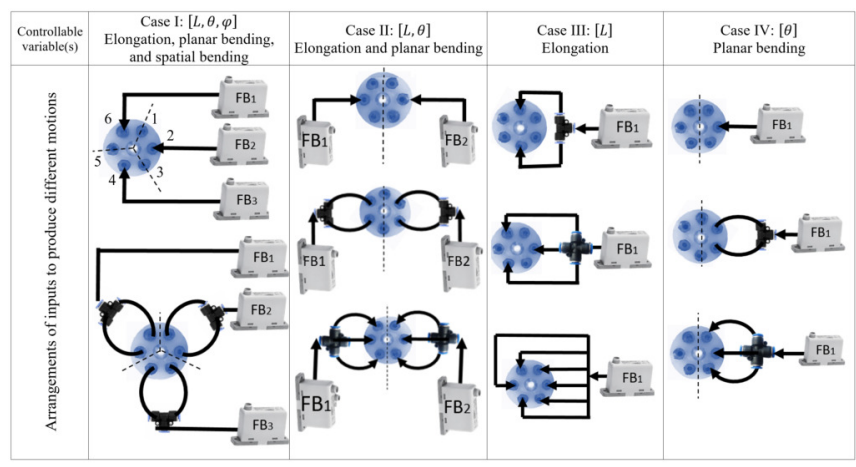

(b)

Fig. 2. (a) Geometric notation, parameters, and chamber arrangement of the robotic manipulator. (b) Chamber air pressure inputs: Case I: Three inputs results in elongation $[L]$, planar bending $[\theta]$, spatial bending $[\phi]$. Case II: Two different, symmetrical pressure inputs results in planar bending $[\theta]$, elongation $[L]$. Case III: A symmetrical pressure input results in elongation $[L]$. Case IV: Pressure input into a semicircle results in planar bending $[\theta]$.

where $m$ is the nominal mass of the robot. Using Taylor expansion, the gravitational potential energy is obtained

$$
U_{G}=m g L \sum_{n=0}^{\infty} \frac{(-1)^{n} \theta^{2 n}}{(2 n) !}
$$

where $g$ denote the acceleration of gravity.

\section{A. Dynamic equations for spatial motion}

Employing equations (5)-(7), the dynamic equations for spatial behaviour can be obtained as

$$
\begin{gathered}
{\left[\begin{array}{ccc}
\zeta_{L} & L \eta_{L} & 0 \\
\zeta_{\theta} & L \eta_{\theta} & 0 \\
0 & 0 & L \eta_{\phi}
\end{array}\right]\left[\begin{array}{c}
\ddot{L} \\
\ddot{\theta} \\
\ddot{\phi}
\end{array}\right]+\left[\begin{array}{c}
-\frac{1}{2} g\left(1-\frac{\theta^{2}}{6}+\frac{\theta^{4}}{120}\right) \\
-\frac{1}{2} g L\left(-\frac{\theta}{3}+\frac{\theta^{3}}{30}\right) \\
0
\end{array}\right]+} \\
{\left[\begin{array}{ccccc}
2 \lambda_{L} & 0 & 0 & L v_{L} & -L \alpha_{L} \\
2 \lambda_{\theta} & 0 & 0 & L v_{\theta} & -L \alpha_{\theta} \\
0 & 2 \lambda_{\phi} & 2 L v_{\phi} & 0 & 0
\end{array}\right]\left[\begin{array}{c}
\dot{L} \dot{\theta} \\
\dot{L} \dot{\phi} \\
\dot{\phi} \dot{\theta} \\
\dot{\theta}^{2} \\
\dot{\phi}^{2}
\end{array}\right]=\frac{1}{m}\left[\begin{array}{c}
Q_{L} \\
Q_{\theta} \\
Q_{\phi}
\end{array}\right]}
\end{gathered}
$$

where,

$$
\begin{aligned}
& \zeta_{L}=\mathscr{A}^{2}+\mathscr{B}^{2}, \eta_{L}=\mathscr{A} \mathscr{C}+\mathscr{B} \mathscr{D}, \lambda_{L}=\mathscr{A} \mathscr{C}+\mathscr{B} \mathscr{D}, v_{L}=\mathscr{A} \mathscr{E}-\mathscr{B} \mathscr{H}, \\
& \alpha_{L}=\mathscr{A}^{2}, \zeta_{\theta}=\mathscr{A} \mathscr{C}+\mathscr{B} \mathscr{D}, \eta_{\theta}=\mathscr{C}^{2}+\mathscr{D}^{2}, \lambda_{\theta}=\mathscr{C}^{2}+\mathscr{D}^{2}, \\
& v_{\theta}=\mathscr{C} \mathscr{Q}+\mathscr{D} \mathscr{H}, \alpha_{\theta}=\mathscr{A} \mathscr{C}, \eta_{\phi}=\mathscr{A}^{2}, \lambda_{\phi}=\mathscr{A}^{2}, v_{\phi}=\mathscr{A} \mathscr{C}
\end{aligned}
$$


and

$$
\begin{aligned}
& \mathscr{A}=\sum_{n=1}^{\infty} \frac{(-1)^{n+1} \theta^{2 n-1}}{(2 n) !}, \mathscr{B}=\sum_{n=0}^{\infty} \frac{(-1)^{n} \theta^{2 n}}{(2 n+1) !}, \mathscr{C}=\sum_{n=1}^{\infty} \frac{(-1)^{n+1} \theta^{2 n-2}}{2 n(2 n-2) !} \\
& \mathscr{D}=\sum_{n=1}^{\infty} \frac{(-1)^{n} \theta^{2 n-1}}{(2 n+1)(2 n-1) !}, \mathscr{E}=\sum_{n=2}^{\infty} \frac{(-1)^{n+1} \theta^{2 n-3}}{2 n(2 n-3) !} \\
& \mathscr{H}=\sum_{n=1}^{\infty} \frac{(-1)^{n} \theta^{2 n-2}}{(2 n+1)(2 n-2) !}, \mathscr{N}=\sum_{n=0}^{\infty} \frac{(-1)^{n} \theta^{2 n+1}}{(2 n+3) !}
\end{aligned}
$$

\section{B. Deriving generalised forces for spatial motion}

Fig. 2(b) (Case I) illustrates the pattern for spatial actuation including elongation $[L]$, planar bending $[\theta]$ and spatial bending $[\phi]$. The generalised force $Q_{j}$ for spatial motion is given by Equation (9).

$$
Q_{j}=\sum_{i=1}^{3}{ }^{w} F_{i} \frac{\partial^{w} r_{i}}{\partial q_{j}}, j=1,2,3
$$

where $q_{j}=[L, \theta, \phi]$, and ${ }^{w} F_{i}(i=1,2,3)$ are the three external forces in the world reference frame when ${ }^{w} F_{i}={ }^{w} T_{h}{ }^{h} F_{i} .{ }^{h} F_{i}$ $(i=1,2,3)$ are the external forces in the body reference frame for ${ }^{h} F_{i}=\left[\begin{array}{lll}0 & 0 & F_{B i}\end{array}\right](i=1,2,3)$.

$$
F_{B i}=P_{i} A_{i}, i=1,2,3
$$

Equation (10) is utilised to identify the forces produced by the air pressures $P_{i}$ in each chamber on the corresponding cross-sectional areas $A_{i}$. The parameters ${ }^{w} r_{i}$ in Equation (9) are the positions of the forces ${ }^{w} F_{i}$ in the world reference frame for ${ }^{w} r_{i}={ }^{w} T_{h}{ }^{h} r_{i},(i=1,2,3)$, where ${ }^{h} r_{1}=\left[\begin{array}{lll}r_{c} & 0 & 0\end{array}\right]^{T}$, ${ }^{h} r_{2}=\left[\begin{array}{lll}-\frac{1}{2} r_{c} & \frac{\sqrt{3}}{2} r_{c} & 0\end{array}\right]^{T}$, and ${ }^{h} r_{3}=\left[\begin{array}{lll}-\frac{1}{2} r_{c} & -\frac{\sqrt{3}}{2} r_{c} & 0\end{array}\right]^{T}$.

Using $\partial^{w} r_{i} / \partial q_{j}$ in Table I, Equation 9, and utilizing the Taylor expansion $\frac{\sin \theta}{\theta}=\sum_{n=0}^{\infty} \frac{(-1)^{n} \theta^{2 n}}{(2 n+1) !}$ and $\frac{\theta-\sin \theta}{\theta^{2}}=$ $\sum_{n=0}^{\infty} \frac{(-1)^{n} \theta^{2 n+1}}{(2 n+3) !}$ to avoid singularity, the generalised forces for the spatial soft fluidic elastomer robot are obtained as:

$$
\begin{aligned}
Q_{1}= & \mathscr{B}\left(F_{B 1}+F_{B 2}+F_{B 3}\right) \\
Q_{2}= & \left(-\cos \phi r_{c}+L \mathscr{N}\right) F_{B 1}+\left(\frac{r_{c}}{2} \cos \phi-\frac{\sqrt{3}}{2} r_{c} \sin \phi+L \mathscr{N}\right) F_{B 2}+ \\
& \left(\frac{r_{c}}{2} \cos \phi+\frac{\sqrt{3}}{2} r_{c} \sin \phi+L \mathscr{N}\right) F_{B 3} \\
Q_{3}= & \left(r_{c} \sin \phi \sin \theta\right) F_{B 1}+\left(-\frac{r_{c}}{2} \sin \phi \sin \theta-\frac{\sqrt{3}}{2} r_{c} \cos \phi \sin \theta\right) F_{B 2}+ \\
& \left(-\frac{r_{c}}{2} \sin \phi \sin \theta+\frac{\sqrt{3}}{2} r_{c} \cos \phi \sin \theta\right) F_{B 3}
\end{aligned}
$$

The visco-elastic property of the material results in forces and momenta described by

$$
\begin{aligned}
& F_{\text {viscoelastic }_{L}}=-F_{\text {elastic }_{L}}-F_{\text {viscous }_{L}} \\
& M_{\text {viscoelastic }_{\theta}}=-M_{\text {elastic }_{\theta}}-M_{\text {viscous }_{\theta}} \\
& M_{\text {viscoelastic }_{\phi}}=-M_{\text {elastic }_{\phi}}-M_{\text {viscous }_{\phi}}
\end{aligned}
$$

where $F_{\text {elastic }_{L}}=k_{L_{e q}} d, F_{\text {viscous }_{L}}=b_{L_{e q}} \dot{d}, M_{\text {elastic }_{\theta}}=k_{\theta_{e q}} \theta$, $M_{\text {viscous }_{\theta}}=b_{\theta_{e q}} \dot{\theta}, M_{\text {elastic }_{\phi}}=k_{\phi_{e q}} \phi$, and $M_{\text {viscous }_{\phi}}=b_{\phi_{e q}} \dot{\phi}$ and parameters $k_{L_{e q}}, b_{L_{e q}}, k_{\theta_{e q}}, b_{\theta_{e q}}, k_{\phi_{e q}}$, and $b_{\phi_{e q}}$ denote the equivalent longitudinal stiffness, longitudinal damping coefficient, planar bending stiffness, planar bending damping coefficient, spatial bending stiffness, and spatial bending damping coefficient. $d$ is the elongation $\left(d=L-L_{0}\right)$ and
TABLE I

GENERALISED FORCES

\begin{tabular}{|c|c|c|c|}
\hline$\partial^{w} r_{i} / \partial q_{j}$ & $\mathbf{j}=\mathbf{1}$ & $\mathbf{j}=\mathbf{2}$ & $\mathbf{j = 3}$ \\
\hline $\mathbf{i}=\mathbf{1}$ & $\frac{\sin \theta}{\theta}$ & $r_{c} \sin \phi \sin \theta$ & $-r_{c} \cos \phi+L \frac{\theta-\sin \theta}{\theta^{2}}$ \\
\hline $\mathbf{i}=\mathbf{2}$ & $\frac{\sin \theta}{\theta}$ & $\frac{-r_{c}}{2} \sin \phi \sin \theta-$ & $\frac{r_{c}}{2} \cos \phi-\frac{\sqrt{3}}{2} r_{c} \sin \phi+$ \\
& & $\frac{\sqrt{3}}{2} r_{c} \cos \phi \sin \theta$ & $L \frac{\theta-\sin \theta}{\theta^{2}}$ \\
\hline $\mathbf{i}=\mathbf{3}$ & $\frac{\sin \theta}{\theta}$ & $\frac{-r_{c}}{2} \sin \phi \sin \theta+$ & $\frac{r_{c}}{2} \cos \phi+\frac{\sqrt{3}}{2} r_{c} \sin \phi+$ \\
& & $\frac{\sqrt{3}}{2} r_{c} \cos \phi \sin \theta$ & $L \frac{\theta-\sin \theta}{\theta^{2}}$ \\
\hline
\end{tabular}

$\dot{d}=\dot{L} . k_{L_{e q}}, b_{L_{e q}}, k_{\theta_{e q}}, b_{\theta_{e q}}$ will be identified in Section V. Thus, $Q_{L}, Q_{\theta}, Q_{\phi}$ in Equation (8) are obtained as follows.

$$
\begin{aligned}
& Q_{L}=Q_{1}+F_{\text {viscoelastic }_{L}} \\
& Q_{\theta}=Q_{2}+M_{\text {viscoelastic }_{\theta}} \\
& Q_{\phi}=Q_{3}+M_{\text {viscoelastic }_{\phi}}
\end{aligned}
$$

\section{Dynamic equations for planar motion}

Fig. 2(b) (Case II) shows the actuation pattern for planar motion which includes planar bending $[\theta]$ and elongation $[L]$ when two different air pressure values are applied to symmetrical chambers. The dynamic equations for planar motion are derived by omitting $\phi, \dot{\phi}, \ddot{\phi}$ from Equation (8). The planar dynamical equations are shown below.

$$
\left[\begin{array}{ll}
\zeta_{L} & L \eta_{L} \\
\zeta_{\theta} & L \eta_{\theta}
\end{array}\right]\left[\begin{array}{c}
\ddot{L} \\
\ddot{\theta}
\end{array}\right]+\left[\begin{array}{cc}
2 \lambda_{L} & L v_{L} \\
2 \lambda_{\theta} & L v_{\theta}
\end{array}\right]\left[\begin{array}{c}
\dot{L} \dot{\theta} \\
\dot{\theta}^{2}
\end{array}\right]+\left[\begin{array}{c}
-\frac{1}{2} g\left(1-\frac{\theta^{2}}{6}+\frac{\theta^{4}}{120}\right) \\
-\frac{1}{2} g L\left(-\frac{\theta}{3}+\frac{\theta^{3}}{30}\right)
\end{array}\right]=\frac{1}{m}\left[\begin{array}{c}
Q_{L} \\
Q_{\theta}
\end{array}\right]
$$

where

$$
\begin{aligned}
\zeta_{L} & =\mathscr{A}^{2}+\mathscr{B}^{2}, \eta_{L}=\mathscr{A} \mathscr{C}+\mathscr{B} \mathscr{D}, \lambda_{L}=\mathscr{A} \mathscr{C}+\mathscr{B} \mathscr{D}, v_{L}=\mathscr{A} \mathscr{E}-\mathscr{B} \mathscr{H}, \\
\zeta_{\theta} & =\mathscr{A} \mathscr{C}+\mathscr{B} \mathscr{D}, \eta_{\theta}=\mathscr{C}^{2}+\mathscr{D}^{2}, \lambda_{\theta}=\mathscr{C}^{2}+\mathscr{D}^{2}, v_{\theta}=\mathscr{C} \mathscr{Q}+\mathscr{D} \mathscr{H}
\end{aligned}
$$

\section{Generalised forces for planar motion}

The generalised forces for planar motion cannot be obtained directly from omitting a single force from Equation (9) since the location, where the forces are applied, is different when compared to the spatial model. The generalised forces $Q_{j}$ for planar movement are instead obtained using $Q_{j}=$ $\sum_{i=1}^{2}{ }^{w} F_{i} \frac{\partial^{w} r_{i}}{\partial q_{j}}(j=1,2)$, where $q_{j}=[L, \theta]$ and ${ }^{w} F_{i}(i=1,2)$ are the two external forces in the world reference frame. Parameters ${ }^{w} r_{1}$ and ${ }^{w} r_{2}$ are obtained for ${ }^{w} r_{i}={ }^{w} T_{h}{ }^{h} r_{i},(i=$ $1,2)$, where ${ }^{h} r_{1}=\left[\begin{array}{lll}r_{c} & 0 & 0\end{array}\right]^{T}$ and ${ }^{h} r_{2}=\left[\begin{array}{lll}-r_{c} & 0 & 0\end{array}\right]^{T}$. Thus, the generalised forces for planar motion are given by:

$$
\begin{aligned}
Q_{1} & =\mathscr{B}\left(F_{B 1}+F_{B 2}\right) \\
Q_{2} & =\left(-r_{c}+L \mathscr{N}\right) F_{B 1}+\left(r_{c}+L \mathscr{N}\right) F_{B 2}
\end{aligned}
$$

\section{EXPERIMENTAL SETUP, VISCO-ELASTIC PARAMETER IDENTIFICATION AND VALIDATION}

\section{A. Experimental setup}

The schematic of the experimental setup is shown in Fig. 3. Pressurised air is provided by a compressor (BAMBI MD Range Model 150/500). Three pressure regulators (Camozzi K8P) are used to pressurise a single (or dual/triple) chamber(s) of the manipulator. The pressure regulators receive control commands from a PC via a DAQ board (NI 


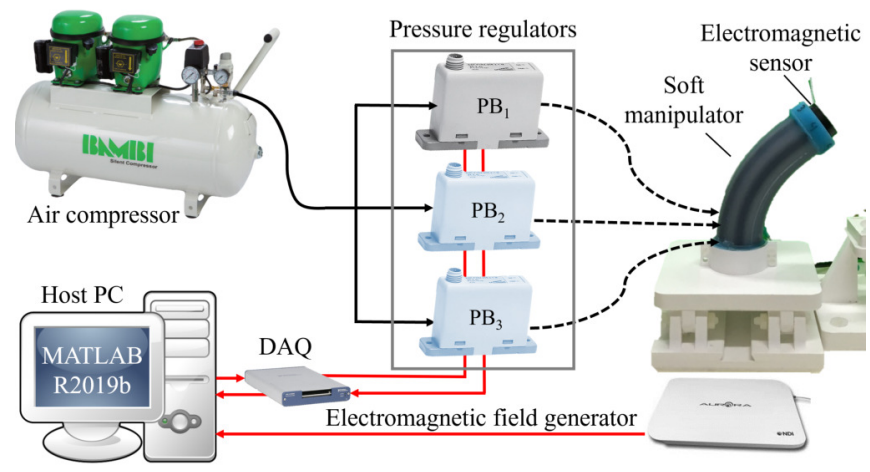

Fig. 3. Setup of the experimental platform.

USB-6341). The tip position of the manipulator is recorded by an NDI Aurora electromagnetic sensor.

\section{B. Experiment 1: Longitudinal stiffness identification}

Three symmetrical chambers are actuated simultaneously with the same pressure (see Fig. 2(b), column 3, row 2) resulting in elongation. The results are shown in Fig. 4 where pressure is plotted versus tip displacement. Values for the calculated external forces using Equation (10) and the robot tip displacements describe the longitudinal stiffness using a Hookean stiffness assumption $F_{\text {elastic }}=k_{L_{e q}} d$. The longitudinal stiffness $k_{L_{e q}}$ is approximately $246 \frac{\mathrm{N}}{\mathrm{m}}$.

\section{Experiment 2: Bending stiffness identification}

To measure the bending stiffness, three chambers on one side of the manipulator were actuated simultaneously with pressure values in the range of [0-1.5] bar. Figs. 5(a) and (b) show $\theta$ and $R_{\text {curvature }}$, obtained from Equation (14). $\theta$ is in the range of $[0,140]$ degrees while the pressure is in the range of $[0,1.5]$ bar. Fig. 5(b) shows that the radius of curvature is [inf, 50] $\mathrm{mm}$ while pressure is in the range of $[0,1.5]$ bar.

$$
\theta=\sin ^{-1}\left(\frac{2 x_{T} z_{T}}{x_{T}^{2}+z_{T}^{2}}\right), R_{\text {curvature }}=\frac{x_{T}^{2}+z_{T}^{2}}{2 x_{T}}
$$

Using the bending moment caused by air pressure $Q_{2}$ for instance in Equation (13), $k_{\theta_{e q}}$ can be obtained (see Fig.5

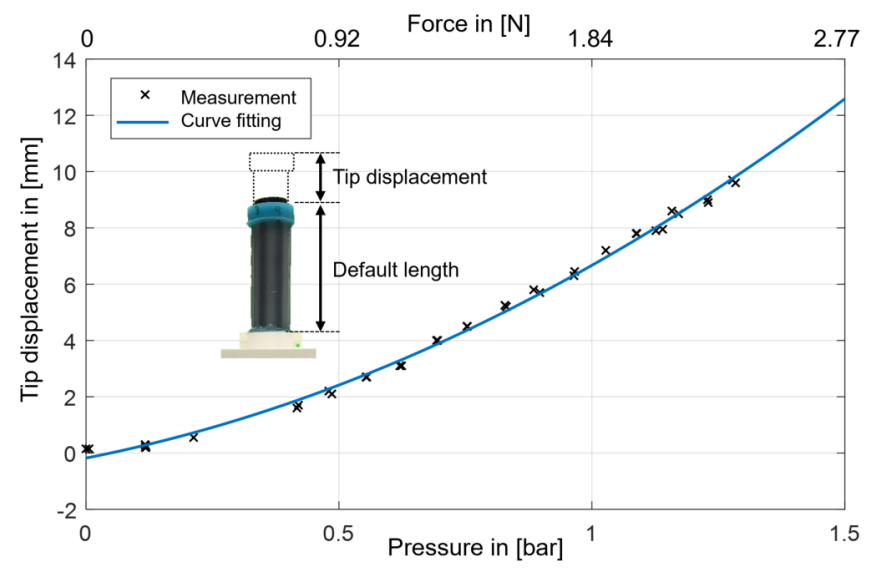

Fig. 4. Experiment 1 results: Tip displacement versus chamber pressures.

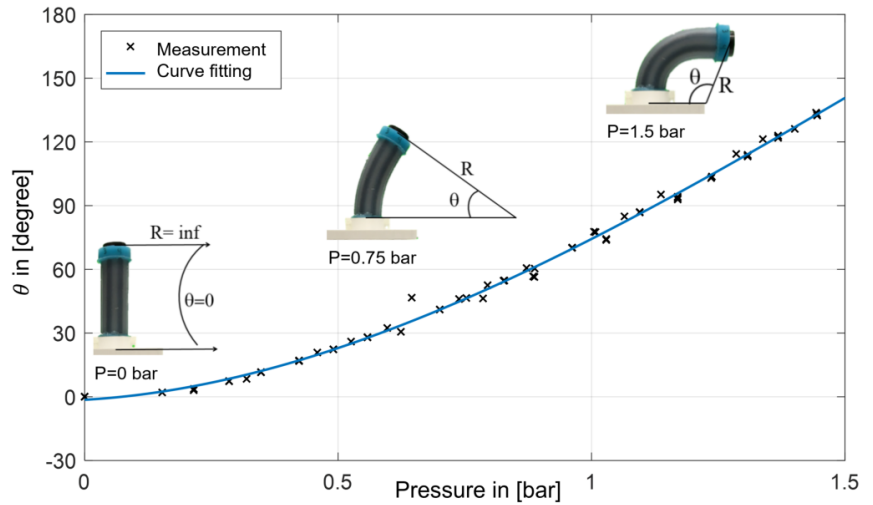

(a)

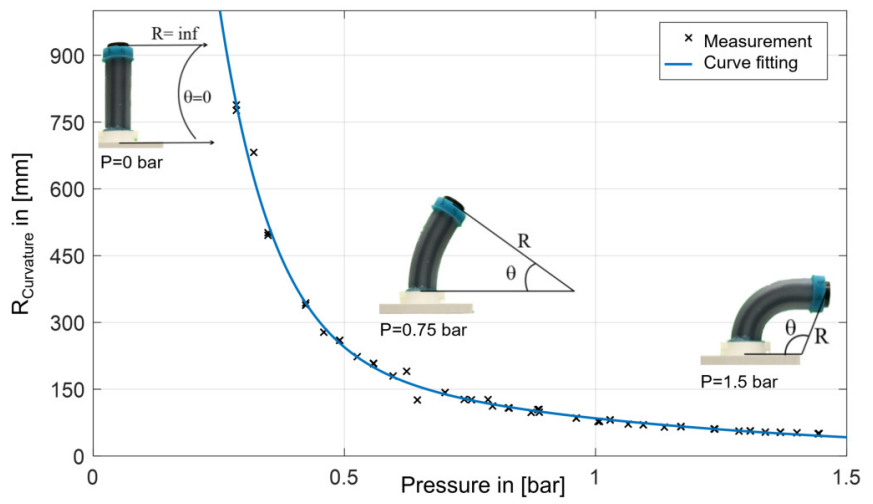

(b)

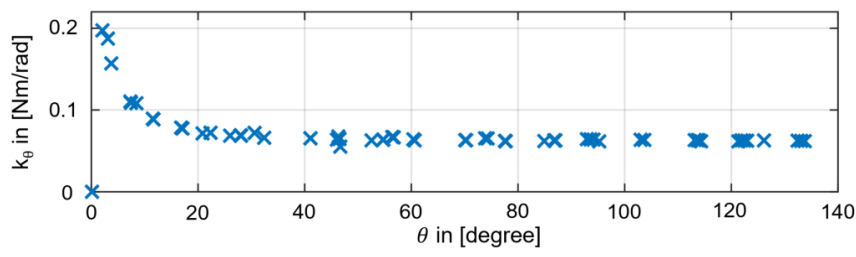

(c)

Fig. 5. Experiment 2 results: (a) The soft robot is actuated with pressure values $[0,1.5]$ bar. The bending angles are measured. (b) Radius of curvature of the soft robot while actuated with the pressure values $[0,1.5]$ bar. (c) Bending stiffness coefficient $\left(k_{\theta}\right)$ versus bending angle $(\theta)$.

(c)). $k_{\theta_{e q}}$ is a nonlinear function and can be approximated to be in the range of $[0.2,0.06] \frac{\mathrm{Nm}}{\mathrm{rad}}$ for a bending angle $\theta$ in the range of $[0,140]$ degrees.

\section{Experiment 3: Longitudinal Damping Identification}

To determine this coefficient, the chambers are simultaneously actuated resulting in longitudinal displacement (see Fig. 6). As the response is overdamped, an exponential curve $Z(t)=A e^{B t}+C e^{D t}, A=-0.003397, B=0.007356$, $C=0.01782, D=-2.833$ is fitted to the time response to assess the damping ratio. The constants $B$ and $D$ are $B=-\omega_{n_{L}} \xi_{L}+\omega_{n_{L}} \sqrt{\xi_{L}^{2}-1}$ and $D=-\omega_{n_{L}} \xi_{L}-\omega_{n_{L}} \sqrt{\xi_{L}^{2}-1}$, where $\xi_{L}=\frac{b_{L_{e q}}}{2 m \omega_{n_{L}}}$ is the damping ratio and $\omega_{n_{L}}=\sqrt{\frac{k_{L e q}}{m}}$ the natural longitudinal frequency. The longitudinal damping coefficient $b_{L_{e q}}$ is approximately $119 \frac{\mathrm{N} . \mathrm{sec}}{\mathrm{m}}$. 


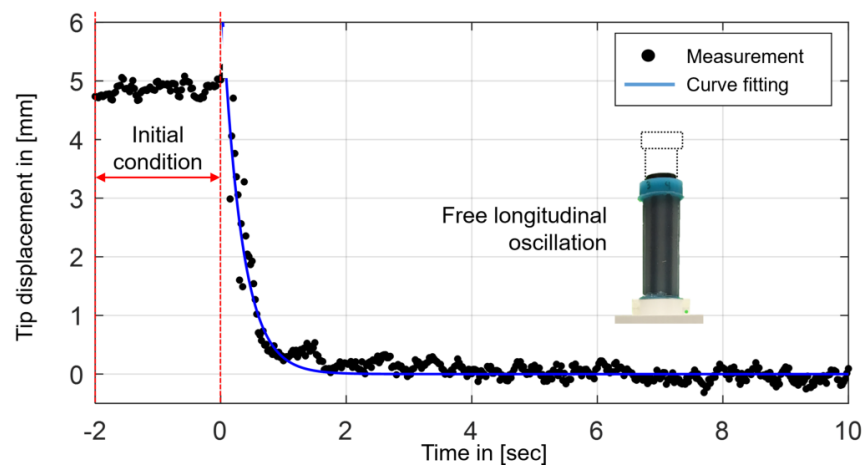

Fig. 6. Experiment 3 results: Example of the soft fluidic elastomer robot's time response to an initial longitudinal displacement.

\section{E. Experiment 4: Bending damping identification}

To calculate the bending damping coefficient, a number of initial planar bending angles are applied to the manipulator and the corresponding time response measured (Fig. 7). The logarithmic decrement $\delta_{\theta}$ is defined as follows:

$$
\delta_{\theta}=\frac{1}{n} \ln \frac{\theta(t)}{\theta(t+n T)}
$$

where $\theta(t)$ is the amplitude at time $t$ and $\theta(t+n T)$ is the amplitude $n$ periods away. The damping ratio of the corresponding bending is obtained as $\xi_{\theta}=\frac{\delta_{\theta}}{\sqrt{4 \pi^{2}+\delta_{\theta}^{2}}}$, and the damping coefficient as $b_{\theta_{e q}}=2 \xi_{\theta} \sqrt{k_{\theta_{e q}} J_{\theta}}$, where $J_{\theta}$ is the rotational mass moment of inertia of the robot around the $\theta$ rotational axis.

\section{F. Experimental validation}

The dynamic model derived in Section IV is validated in experiments comparing the time response of the physical robot with the output of the simulation model based on the experimentally identified parameters. The identified parameters used in the simulation are $k_{L_{e q}}=246 \frac{\mathrm{N}}{\mathrm{m}}, b_{L_{e q}}=119 \frac{\mathrm{Ns}}{\mathrm{m}}$, $k_{\theta_{e q}}=0.06 \frac{\mathrm{Nm}}{\mathrm{rad}}$, and $b_{\theta_{e q}}=0.02 \frac{\mathrm{Nms}}{\mathrm{rad}}$.

The results of two set of experiments are shown in Figs. 8 and 9. Pressure measurements were recorded with $0.1 \mathrm{~ms}$ and positions were recorded with $25 \mathrm{~ms}$ sampling time. In the first set of experiments, six pressure inputs in the

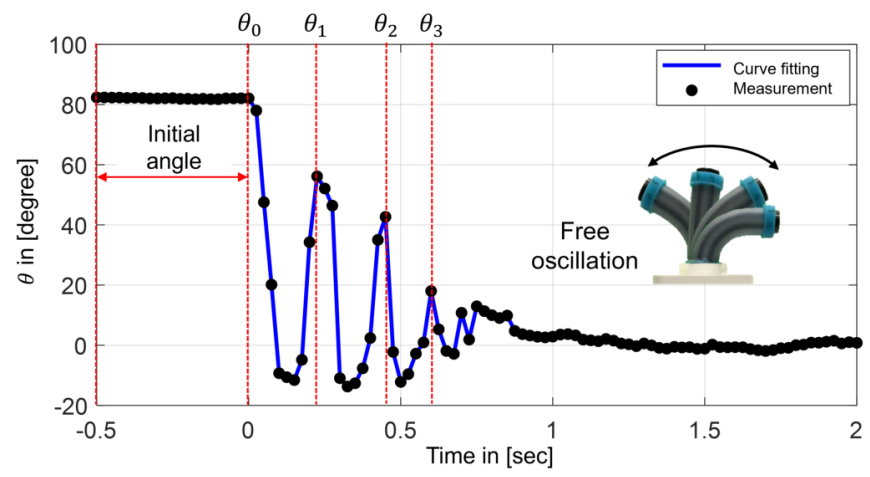

Fig. 7. Experiment 4 results: Soft fluidic elastomer robot time response to an initial bending angle.
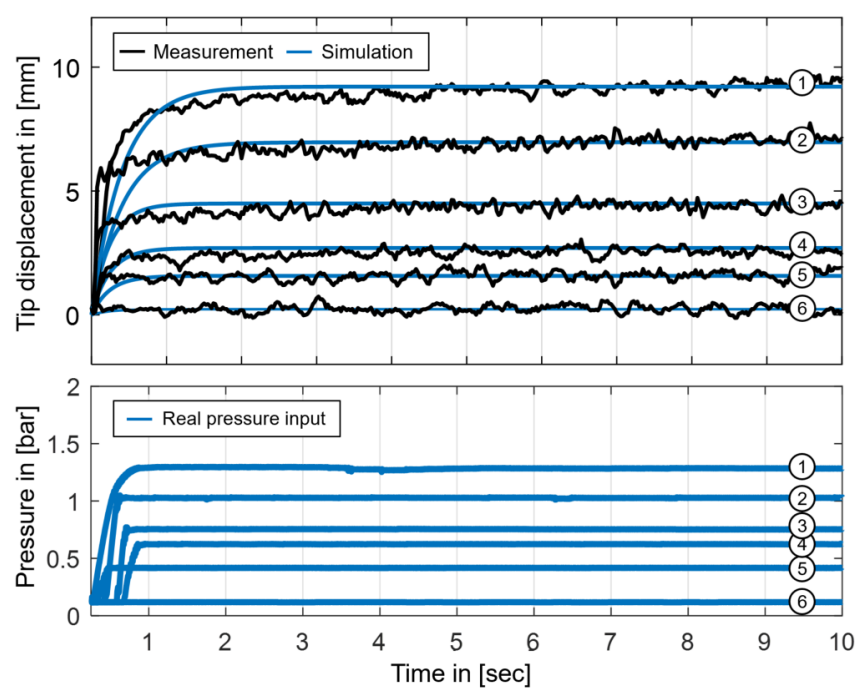

Fig. 8. Time response of the open loop dynamical simulation and real experiment and the corresponding input pressures Pressure is applied to Chambers 1,3, and 5 (Fig. 2, Case III, row 2).

range of $[0.1-1.3]$ bar were applied to Chambers 1,3 and 5 simultaneously (see Fig. 2, Case III, row 2). The pressure inputs applied to the simulated model were the same as the actual step inputs applied to the physical robot. Pure elongation was produced and the corresponding time response (vertical tip displacement) is plotted for both the physical experiments and simulation in Fig. 8. The results validate the accuracy of the identified longitudinal viscoelastic parameters, i.e., $k_{L_{e q}}$ and $b_{L_{e q}}$. In the second set of experiments, six pressure inputs in the range of $[0.1-1.3]$ bar were applied to Chambers 1, 2 and 3 simultaneously (see Fig. 2, Case IV, row 3). The pressures inputs were the same
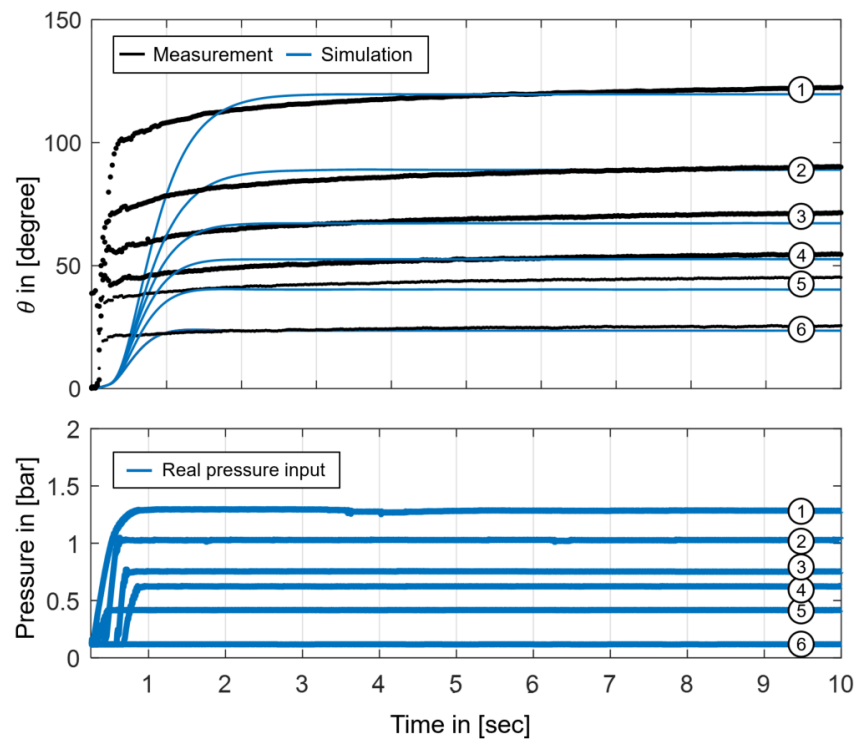

Fig. 9. Time response of the open loop dynamical simulation versus real experiment and the corresponding input pressures. Pressure is applied to Chambers 1,2, and 3 (Fig. 2, Case IV, row 3). 
for both the simulation and physical experiments as shown in Fig. 9. The time response of the system is plotted for the physical experiments and simulation in Fig. 9 validating the bending visco-elastic parameters, i.e., $k_{\theta_{e q}}$ and $b_{\theta_{e q}}$.

\section{CONCLUSION AND FUTURE WORK}

A dynamic model of a fibre-reinforced soft fluidic elastomer manipulator has been developed and experimentally verified. The fibre reinforced non-inflatable fluidic soft robot used in this research can produce both planar and spatial movement and consequently the dynamic equations are developed for both cases. The parameters of the visco-elastic behaviour of the robot have been experimentally determined. The resulting model is validated by comparing the real-time response of the robot with simulation results.

In future work, we will focus on determining the viscoelastic parameters related to spatial bending and spatial dynamic equations. Furthermore, the dynamic equations obtained in this paper will be used to design a nonlinear modelbased closed-loop controller.

\section{REFERENCES}

[1] J. Fras, Y. Noh, M. Macias, H. Wurdemann, and K. Althoefer, "Bioinspired octopus robot based on novel soft fluidic actuator," in IEEE Int Conf Robot Autom, pp. 1583-1588, 2018.

[2] M. W. Hannan and I. D. Walker, "Kinematics and the implementation of an elephant's trunk manipulator and other continuum style robots," Journal of robotic systems, vol. 20, no. 2, pp. 45-63, 2003.

[3] I. A. Gravagne and I. D. Walker, "Uniform regulation of a multisection continuum manipulator," in IEEE Int Conf Robot Autom, vol. 2, pp. 1519-1524, 2002.

[4] A. D. Kapadia, I. D. Walker, D. M. Dawson, and E. Tatlicioglu, "A model-based sliding mode controller for extensible continuum robots," in Int Conf on Signal Process Robot Autom, pp. 113-120, 2010.

[5] H. Feng, Y. Sun, P. A. Todd, and H. P. Lee, "Body wave generation for anguilliform locomotion using a fiber-reinforced soft fluidic elastomer actuator array toward the development of the eel-inspired underwater soft robot," Soft Robotics, vol. 7, no. 2, pp. 233-250, 2020.

[6] S. Kim, C. Laschi, and B. Trimmer, "Soft robotics: a bioinspired evolution in robotics," Trends Biotechnol, vol. 31, no. 5, pp. 287-294, 2013.

[7] R. J. Webster III and B. A. Jones, "Design and kinematic modeling of constant curvature continuum robots: A review," Int J Robot Res, vol. 29, no. 13, pp. 1661-1683, 2010.

[8] A. D. Marchese and D. Rus, "Design, kinematics, and control of a soft spatial fluidic elastomer manipulator," Int J Robot Res, vol. 35, no. 7, pp. 840-869, 2016.

[9] A. Kapadia and I. D. Walker, "Task-space control of extensible continuum manipulators," in IEEE/RSJ Int Conf Intell Robots Syst, pp. 1087-1092, 2011.

[10] A. Amouri, A. Zaatri, and C. Mahfoudi, "Dynamic modeling of a class of continuum manipulators in fixed orientation," Journal of Intelligent \& Robotic Systems, vol. 91, no. 3-4, pp. 413-424, 2018.

[11] A. Amouri, C. Mahfoudi, and A. Zaatri, "Dynamic modeling of a spatial cable-driven continuum robot using euler-lagrange method," Int J Eng Technol, vol. 10, no. 1, p. 60, 2019.

[12] S. Bauer, S. Bauer-Gogonea, I. Graz, M. Kaltenbrunner, C. Keplinger, and R. Schwödiauer, "A soft future: from robots and sensor skin to energy harvesters," Advanced Materials, vol. 26, p. 149-162, 2014.

[13] H. A. Wurdemann, A. Stilli, and K. Althoefer, "Lecture notes in computer science: An antagonistic actuation technique for simultaneous stiffness and position control," in Intelligent Robotics and Applications, pp. 164-174, Springer Int Publishing, 2015.

[14] A. Stilli, A. Cremoni, M. Bianchi, A. Ridolfi, F. Gerii, F. Vannetti, H. A. Wurdemann, B. Allotta, and K. Althoefer, "Airexglove - a novel pneumatic exoskeleton glove for adaptive hand rehabilitation in post-stroke patients," in IEEE Int Conf Soft Robot, pp. 579-584, 2018.
[15] J. M. Gandarias, Y. Wang, A. Stilli, A. J. García-Cerezo, J. M. Gómezde Gabriel, and H. A. Wurdemann, "Open-loop position control in collaborative, modular variable-stiffness-link (VSL) robots," IEEE Robot Autom Lett, vol. 5, no. 2, pp. 1772-1779, 2020.

[16] A. Palombi, G. M. Bosi, S. D. Giuseppe, E. De Momi, S. HomerVanniasinkam, G. Burriesci, and H. A. Wurdemann, "Sizing the aortic annulus with a robotised, commercially available soft balloon catheter: in vitro study on idealised phantoms," in IEEE Int Conf Robot Autom, pp. 6230-6236, 2019.

[17] C. Della Santina, A. Bicchi, and D. Rus, "On an improved state parametrization for soft robots with piecewise constant curvature and its use in model based control," IEEE Robot Autom Lett, vol. 5, no. 2, pp. 1001-1008, 2020.

[18] A. Stilli, E. Kolokotronis, J. Fraś, A. Ataka, K. Althoefer, and H. Wurdemann, "Static kinematics for an antagonistically actuated robot based on a beam-mechanics-based model," in IEEE/RSJ Int Conf Intell Robots Syst, pp. 6959-6964, 2018.

[19] A. Shiva, S. H. Sadati, Y. Noh, J. Fraś, A. Ataka, H. Würdemann, H. Hauser, I. D. Walker, T. Nanayakkara, and K. Althoefer, "Elasticity versus hyperelasticity considerations in quasistatic modeling of a soft finger-like robotic appendage for real-time position and force estimation," Soft Robotics, vol. 6, no. 2, pp. 228-249, 2019.

[20] T. G. Thuruthel, E. Falotico, F. Renda, and C. Laschi, "Learning dynamic models for open loop predictive control of soft robotic manipulators," Bioinspir Biomim, vol. 12, no. 6, p. 066003, 2017.

[21] T. G. Thuruthel, E. Falotico, F. Renda, and C. Laschi, "Model-based reinforcement learning for closed-loop dynamic control of soft robotic manipulators," IEEE Trans Robot, vol. 35, no. 1, pp. 124-134, 2018.

[22] V. Falkenhahn, T. Mahl, A. Hildebrandt, R. Neumann, and O. Sawodny, "Dynamic modeling of constant curvature continuum robots using the euler-lagrange formalism," in IEEE/RSJ Int Conf Intell Robots Syst, pp. 2428-2433, 2014.

[23] V. Falkenhahn, T. Mahl, A. Hildebrandt, R. Neumann, and O. Sawodny, "Dynamic modeling of bellows-actuated continuum robots using the euler-lagrange formalism," IEEE Trans Robot, vol. 31, no. 6, pp. 1483-1496, 2015.

[24] V. Falkenhahn, A. Hildebrandt, R. Neumann, and O. Sawodny, "Dynamic control of the bionic handling assistant," IEEE ASME Trans Mechatron, vol. 22, no. 1, pp. 6-17, 2016.

[25] V. Falkenhahn, A. Hildebrandt, R. Neumann, and O. Sawodny, "Model-based feedforward position control of constant curvature continuum robots using feedback linearization," in IEEE Int Conf Robot Autom, pp. 762-767, 2015.

[26] A. D. Marchese, R. Tedrake, and D. Rus, "Dynamics and trajectory optimization for a soft spatial fluidic elastomer manipulator," Int $J$ Robot Res, vol. 35, no. 8, pp. 1000-1019, 2016.

[27] S. H. Sadati, S. E. Naghibi, I. D. Walker, K. Althoefer, and T. Nanayakkara, "Control space reduction and real-time accurate modeling of continuum manipulators using ritz and ritz-galerkin methods," IEEE Robot Autom Lett, vol. 3, no. 1, pp. 328-335, 2017.

[28] S. M. Mustaza, Y. Elsayed, C. Lekakou, C. Saaj, and J. Fras, "Dynamic modeling of fiber-reinforced soft manipulator: A visco-hyperelastic material-based continuum mechanics approach," Soft robotics, vol. 6, no. 3, pp. 305-317, 2019.

[29] C. Della Santina, R. K. Katzschmann, A. Biechi, and D. Rus, "Dynamic control of soft robots interacting with the environment," in IEEE Int Conf Soft Robot, pp. 46-53, 2018.

[30] R. K. Katzschmann, C. Della Santina, Y. Toshimitsu, A. Bicchi, and D. Rus, "Dynamic motion control of multi-segment soft robots using piecewise constant curvature matched with an augmented rigid body model," in IEEE Int Conf Soft Robot, pp. 454-461, 2019.

[31] C. D. Onal and D. Rus, "Autonomous undulatory serpentine locomotion utilizing body dynamics of a fluidic soft robot," Bioinspir Biomim, vol. 8, no. 2, p. 026003, 2013.

[32] S. M. Mustaza, Modelling and control of a flexible soft robotic uterine elevator. $\mathrm{PhD}$ thesis, University of Surrey, 2018.

[33] J. Fraś, J. Czarnowski, M. Maciaś, J. Główka, M. Cianchetti, and A. Menciassi, "New STIFF-FLOP module construction idea for improved actuation and sensing," in IEEE Int Conf Robot Autom, pp. 2901-2906, 2015.

[34] I. S. Godage, D. T. Branson, E. Guglielmino, G. A. Medrano-Cerda, and D. G. Caldwell, "Shape function-based kinematics and dynamics for variable length continuum robotic arms," in IEEE Int Conf Robot Autom, pp. 452-457, 2011. 